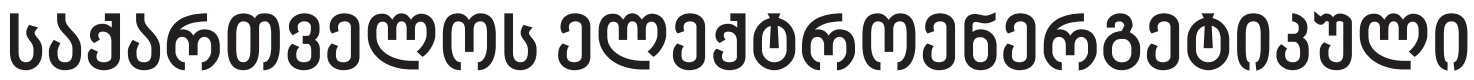

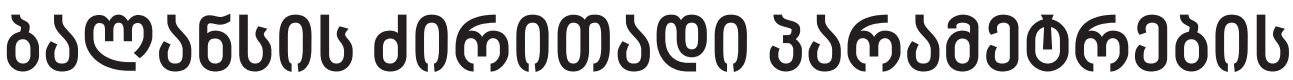

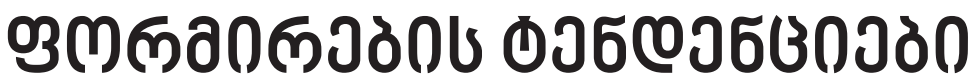

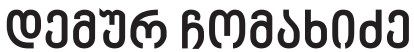

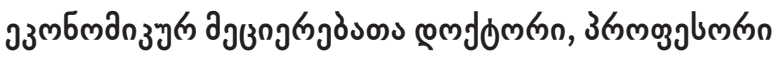 \\ bufuknos $39 \mathrm{mml}$ o oj

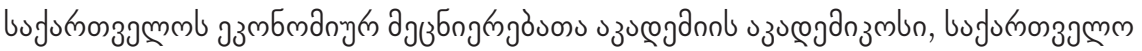 \\ demurchomakhidze@yahoo.com
}

\section{งอ0อ3১ธ Зb১3১0১}

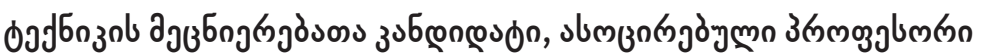

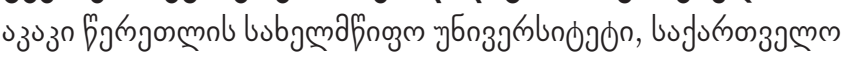

ketevan.tskhakaia@gmail.com

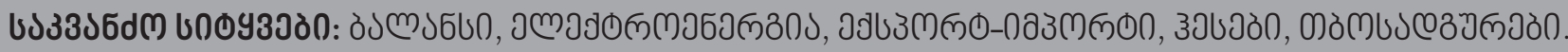

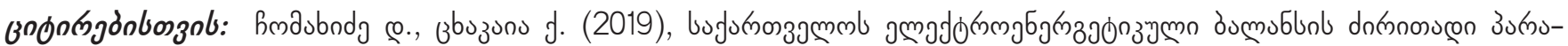
a jom

\section{ฆอง১З১ตั0}

jмaปd

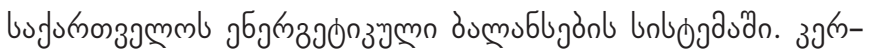

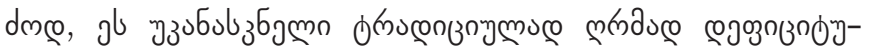

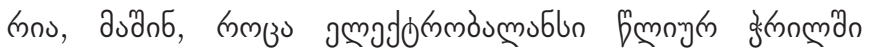

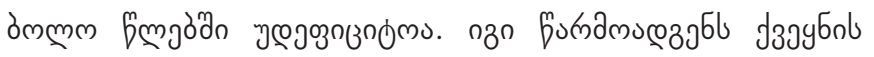

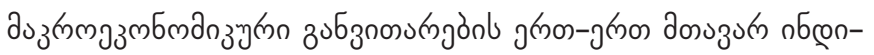

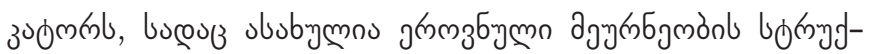

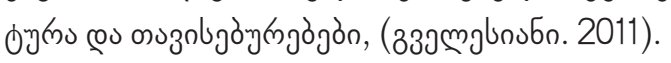

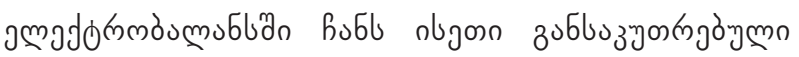

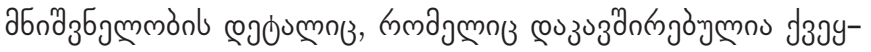

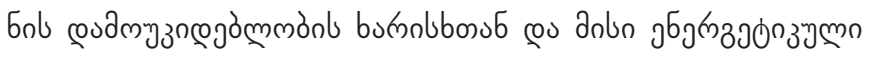

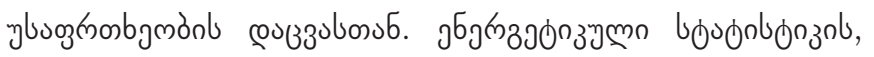

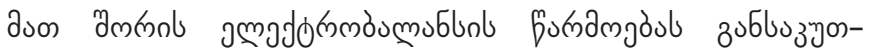

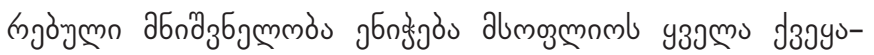
бuдn, anybje

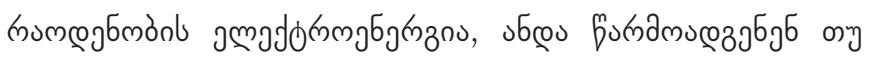

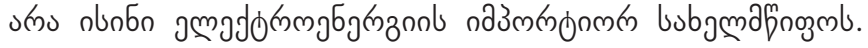

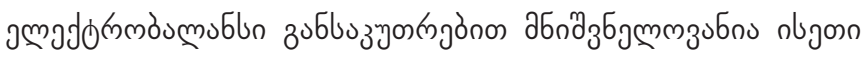

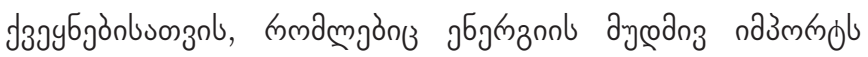

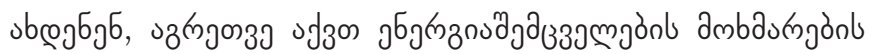

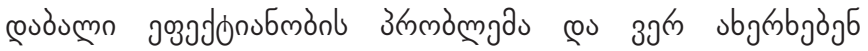

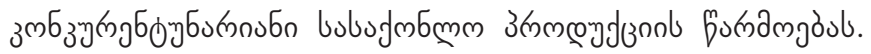
uмб

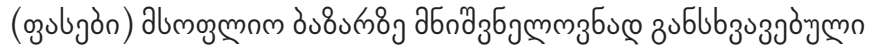

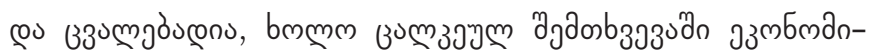

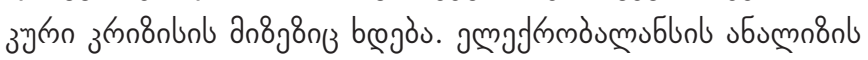

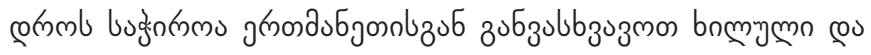

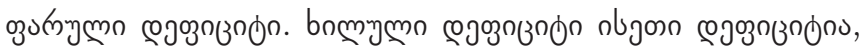

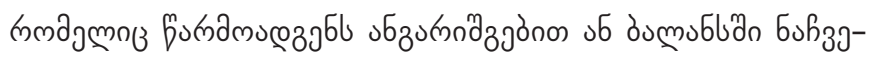

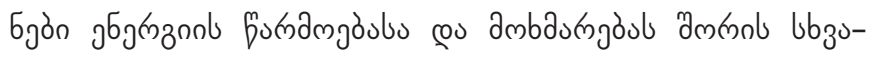

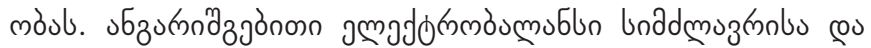

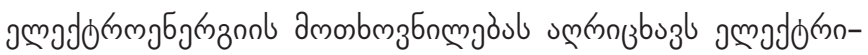

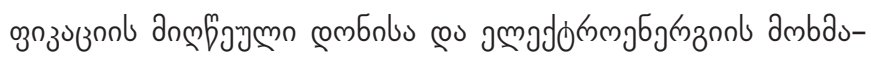

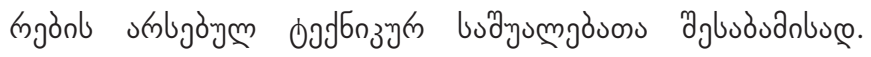




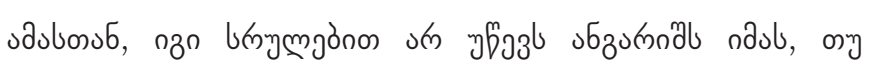

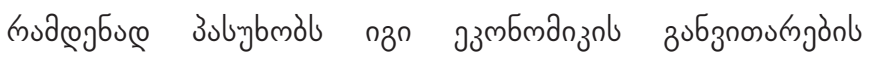

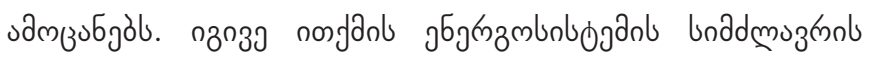

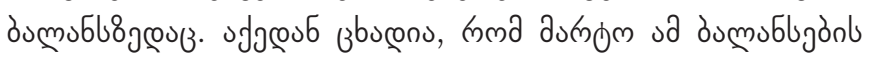

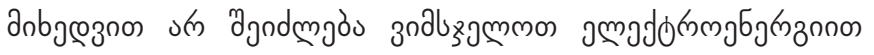

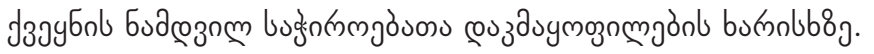

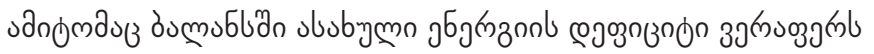

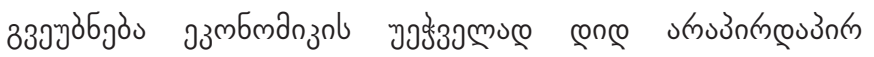

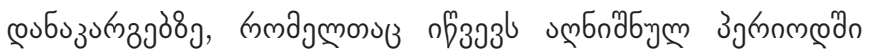

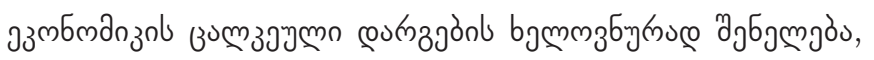

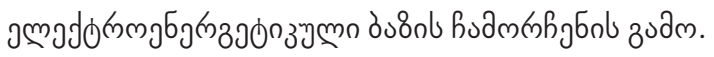

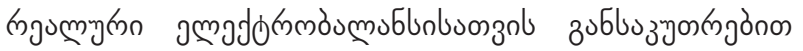

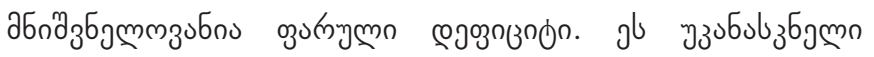

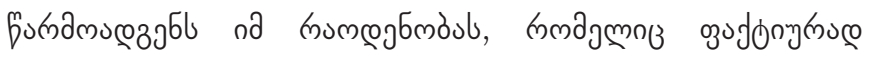

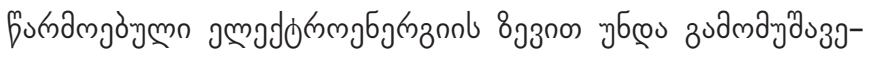

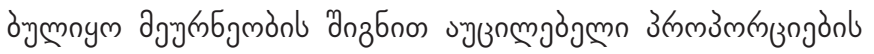

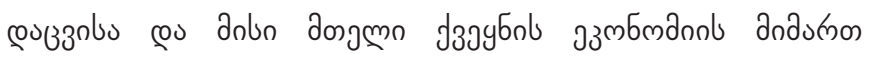

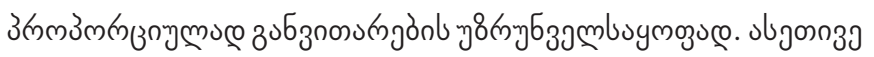

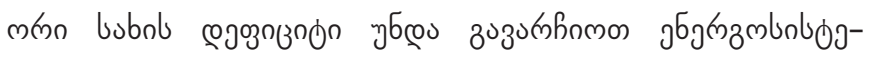

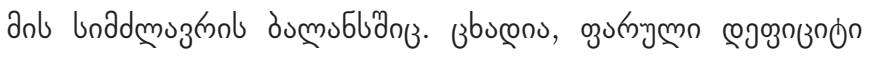

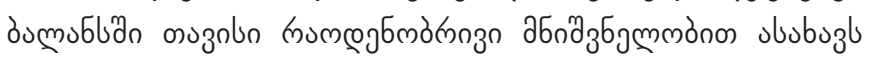

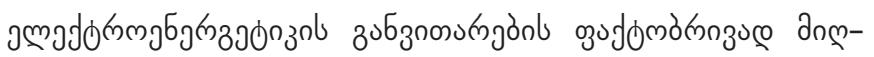

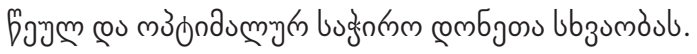

\section{d0m00งео б১60ल0}

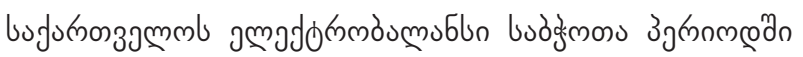

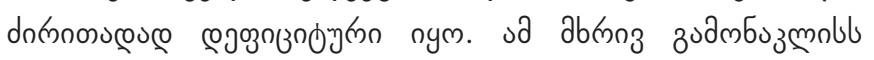

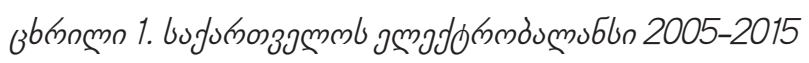

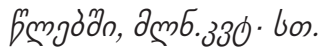

\begin{tabular}{|c|c|c|c|c|c|}
\hline \multirow{2}{*}{ coubsbymgös } & \multicolumn{5}{|c|}{ famn } \\
\hline & 2005 & 2010 & 2012 & 2014 & 2015 \\
\hline 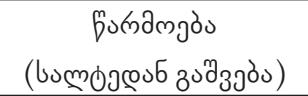 & 6880,8 & 9919,2 & 9471,9 & 10153,7 & 710832,6 \\
\hline àm dmbnt & & & & & \\
\hline 3jon & 5850,2 & 9263,3 & 7122,1 & 8221,1 & 8453,8 \\
\hline का ח & 1030,6 & 655,9 & 2349,8 & 1932,6 & 2378,7 \\
\hline пазмnका & 1398,6 & 222,1 & 614,6 & 851,9 & 699,2 \\
\hline 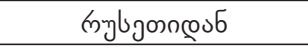 & 615,7 & 211,9 & 517,05 & 665,6 & 511,0 \\
\hline 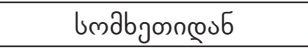 & 252,9 & & & 2,1 & 86,5 \\
\hline 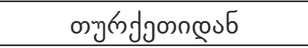 & 9,3 & 0,0001 & 629,0 & - & - \\
\hline 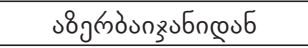 & 20,7 & 4 & 97,54 & 184,2 & 101,7 \\
\hline गd3mகnon & 121,8 & 1524,2 & 528,2 & 603,6 & 659,9 \\
\hline 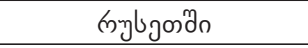 & - & 1117 & 369,43 & 218,6 & 169,6 \\
\hline 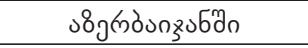 & - & 14,31 & 11,79 & 8,0 & - \\
\hline ๓चங் & 121,5 & 303,4 & 79,0 & 236,5 & 419,4 \\
\hline bmabgoñn & - & 89,5 & 67,9 & 140,5 & 70,9 \\
\hline 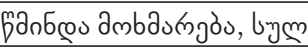 & 7842,2 & 8422,0 & 9379,4 & 10402,0 & 10631,8 \\
\hline dumsoblon $( \pm)$ & $-96,2$ & $+1477,2$ & $+92,5$ & $-248,3$ & $+200,8$ \\
\hline
\end{tabular}

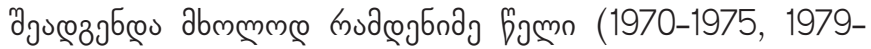

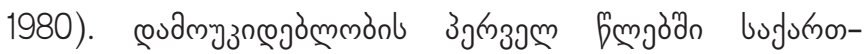

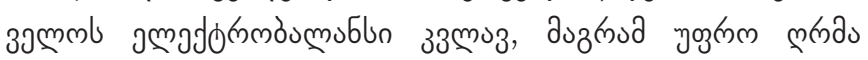

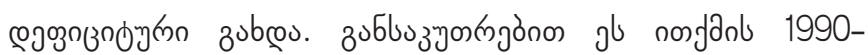

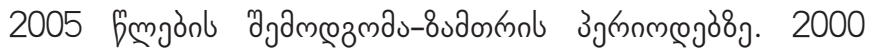

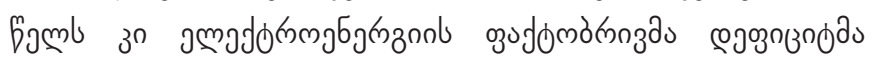

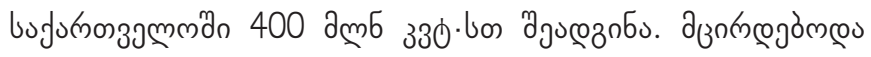

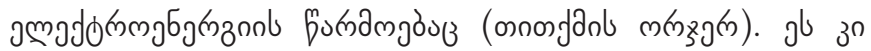

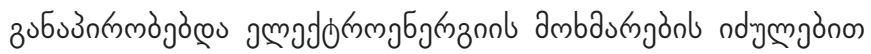

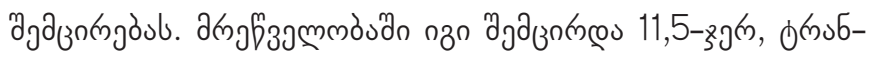

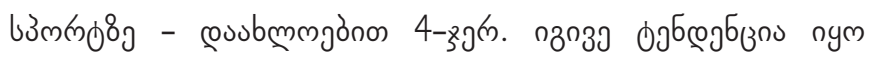

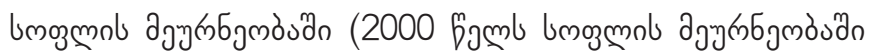

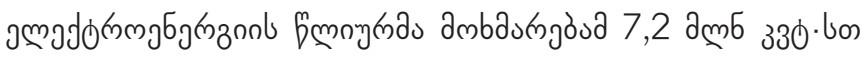

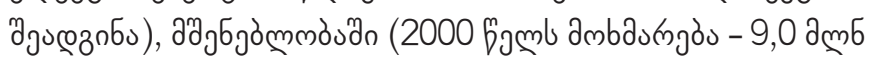

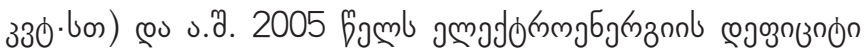

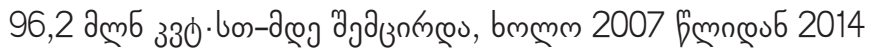

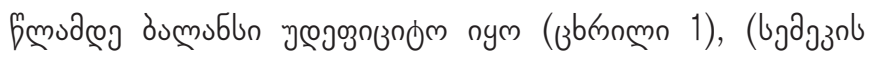

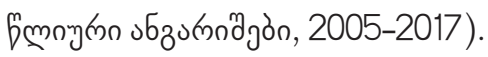

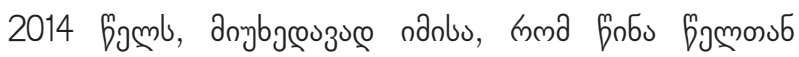

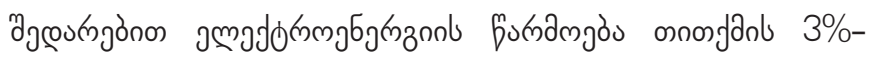

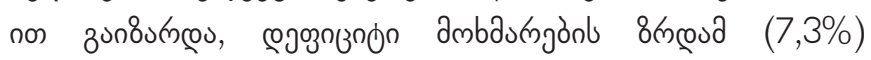

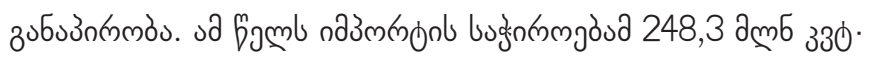

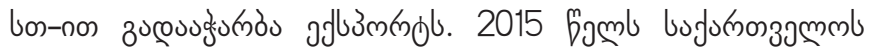

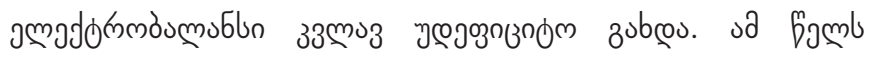

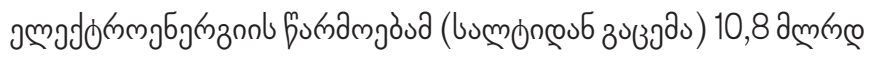

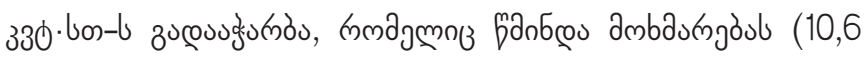
a

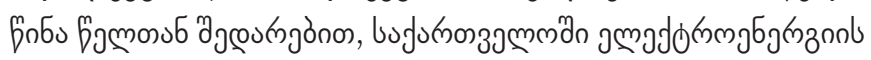

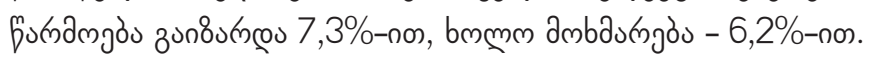

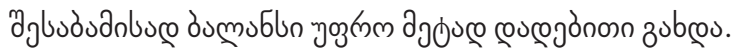

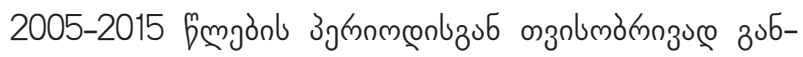

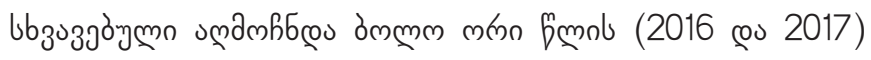

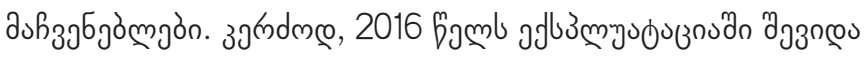

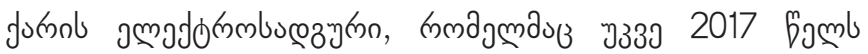

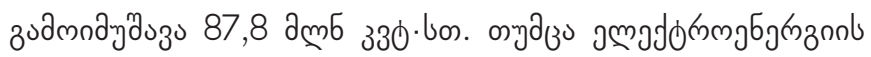

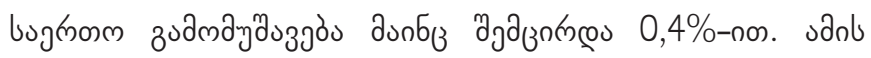

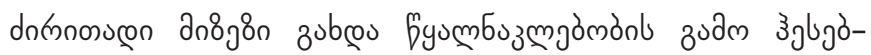

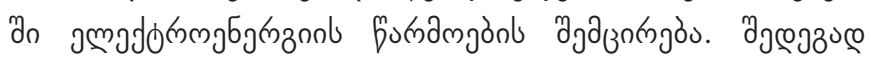

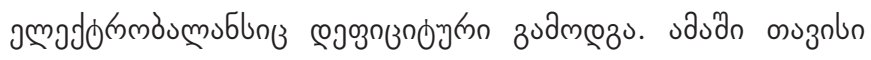

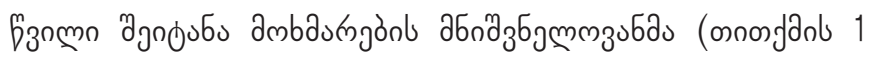

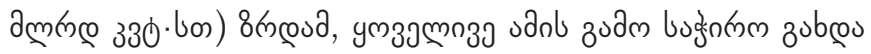

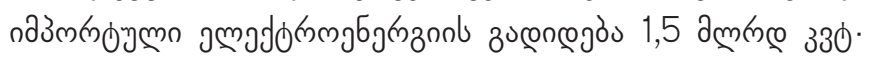

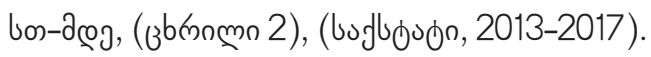

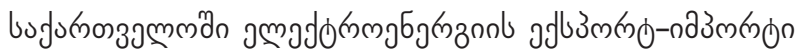

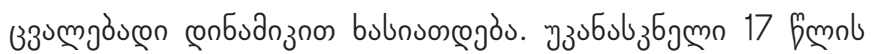

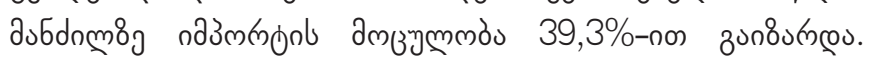

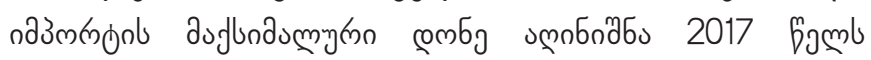




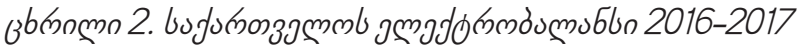

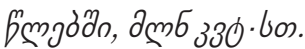

\begin{tabular}{|c|c|c|}
\hline \multirow{2}{*}{ 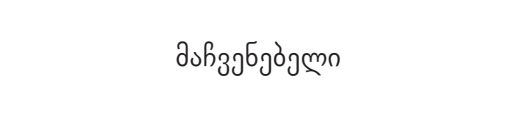 } & \multicolumn{2}{|c|}{ fomo } \\
\hline & 2016 & 2017 \\
\hline 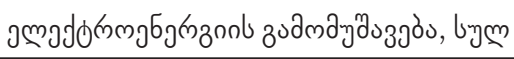 & 11573,6 & 11531,2 \\
\hline \multicolumn{3}{|l|}{ Juon Jmmnll: } \\
\hline 3 an & 9329,2 & 9210,4 \\
\hline oglo & 2235,4 & 2233,0 \\
\hline 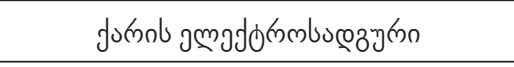 & 9,0 & 87,8 \\
\hline nдзмкnon, lym & 478,9 & 1497,2 \\
\hline aflümmon, bym & 559,0 & 685,7 \\
\hline ambasmjòs & 11493,5 & 12342,7 \\
\hline dumúblon $( \pm)$ & $+80,1$ & $-811,5$ \\
\hline
\end{tabular}

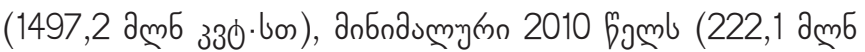

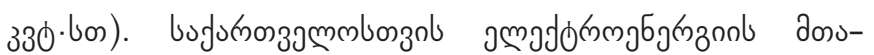

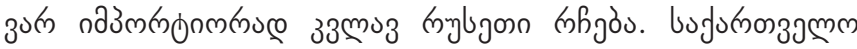

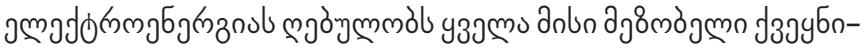

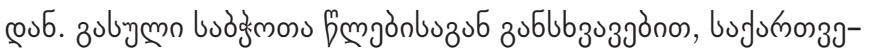

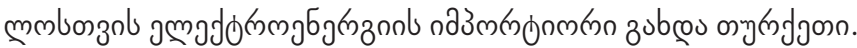

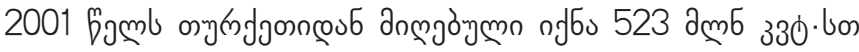

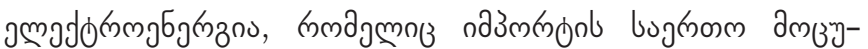

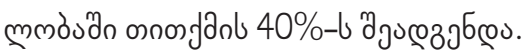

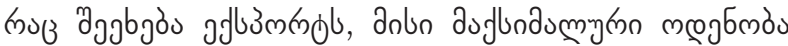

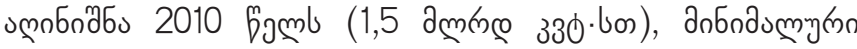

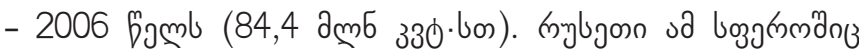

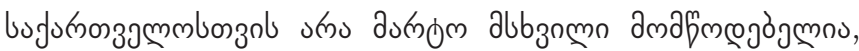

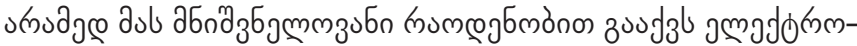

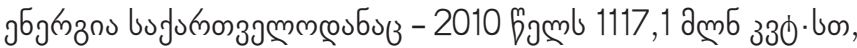

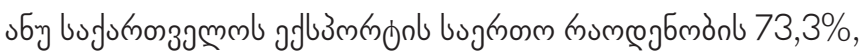
2014 Gamb - 218,6 am 330. bon, งбฑ 36,2\%.

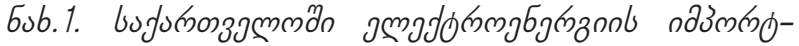
adlusmononb bonydoynd, 2017 p.

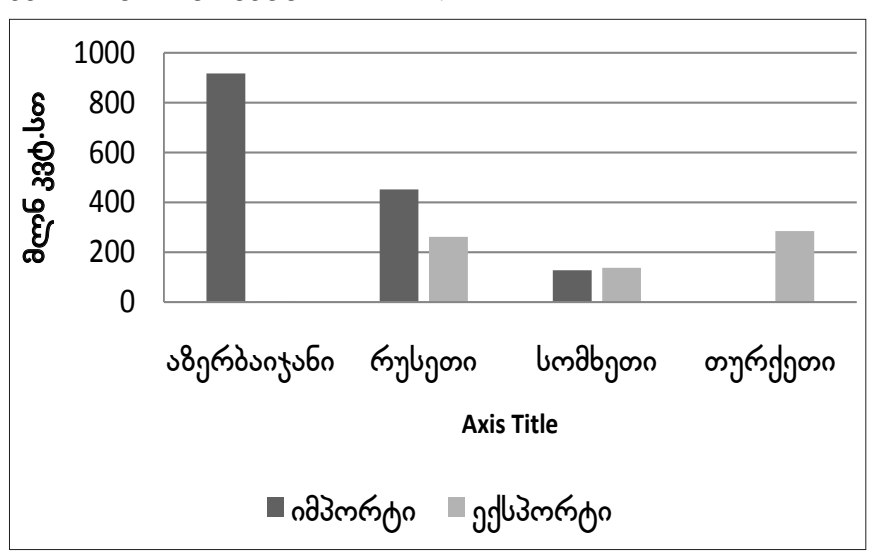

2017 famb amadd

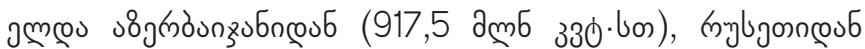

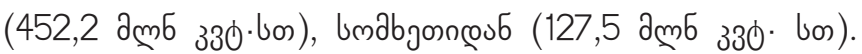

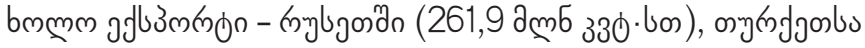

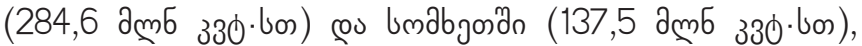

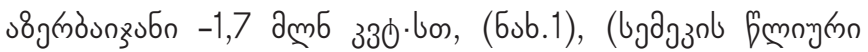

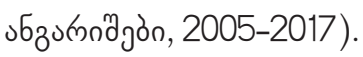

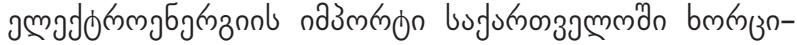

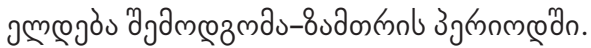

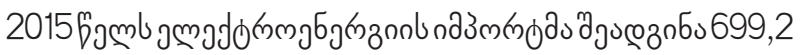
a

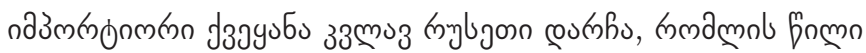

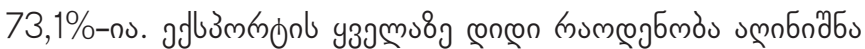

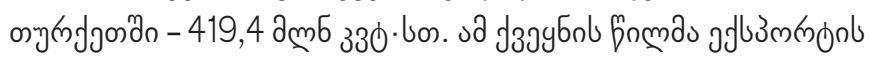

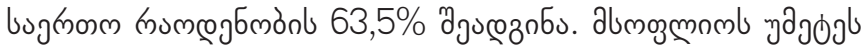

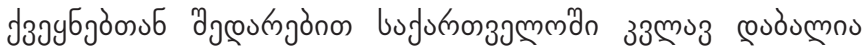

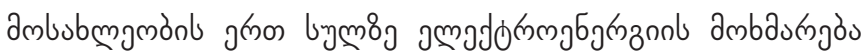
(zbrnnmn 3), (Key World Energy Statistics, 2016).

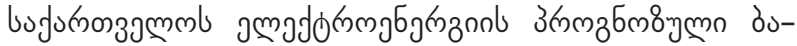

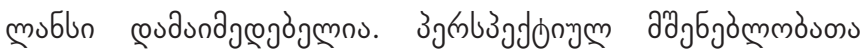

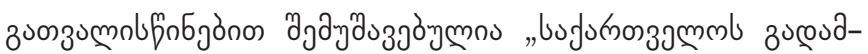

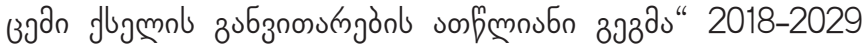

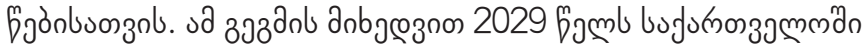

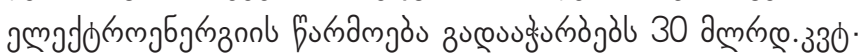

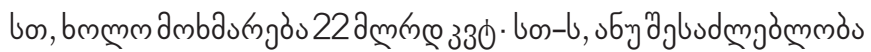

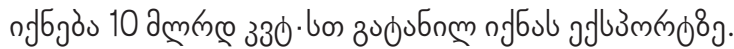

3btromano 3.

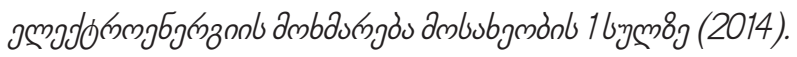

\begin{tabular}{|c|c|c|c|}
\hline dзృуงбo & $\begin{array}{c}\text { ambsbmgmò, } \\
\text { дলmb }\end{array}$ & 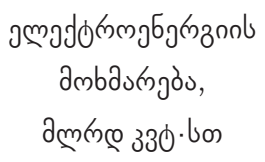 & 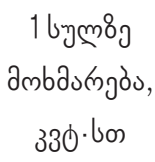 \\
\hline alımoुलnलm & 7249 & 21963 & 3029,8 \\
\hline د $2 \nexists$ & 319,17 & 4137,1 & 12962 \\
\hline (x) & 143,82 & 949,59 & 6602,6 \\
\hline 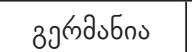 & 80,98 & 569,75 & 7035,7 \\
\hline no3̈mbno & 127,12 & 995,26 & 7829,3 \\
\hline bsogñbagyon & 66,17 & 460,2 & 6954,8 \\
\hline mod3no & 1,99 & 6,9 & 3460 \\
\hline mond & 2,93 & 11,21 & 3820 \\
\hline 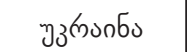 & 45,38 & 154,77 & 3410 \\
\hline bufunonsgmm & 4,5 & 10,02 & 2226,6 \\
\hline 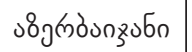 & 9,54 & 21,0 & 2201 \\
\hline lıabjon & 3,01 & 5,71 & 1897 \\
\hline
\end{tabular}




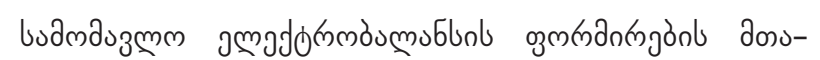

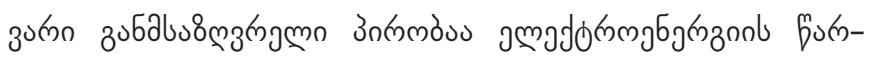

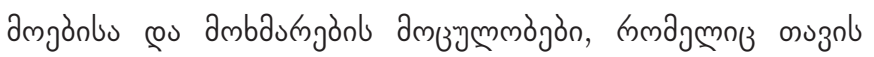

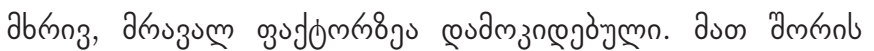

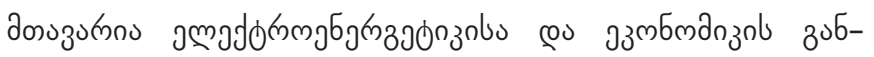

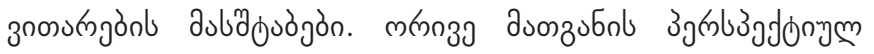

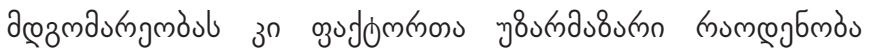
zublus 8 लुз mngòn (hydropower, thermal power, renewable power

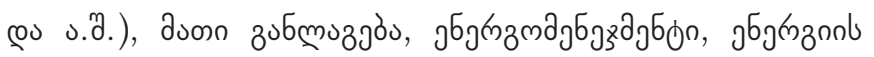

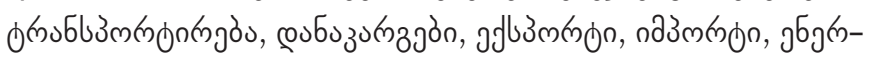

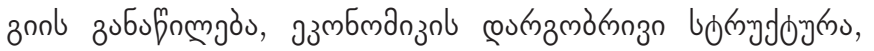

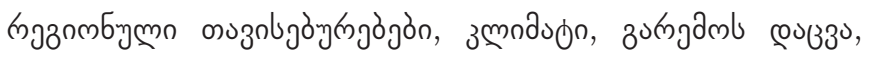

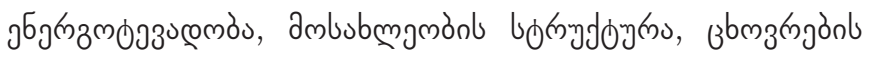

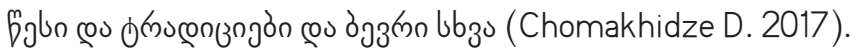

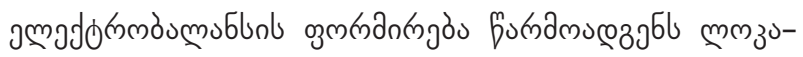

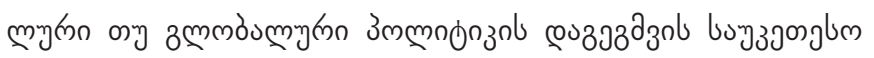

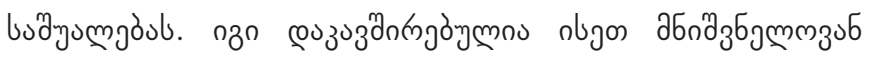

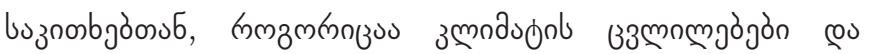

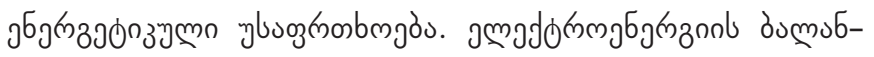

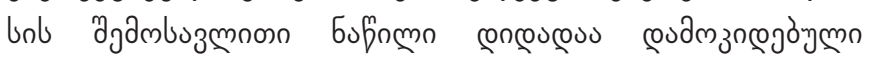

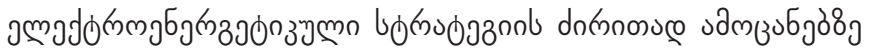

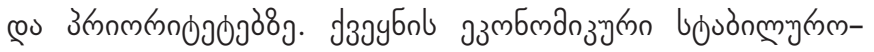

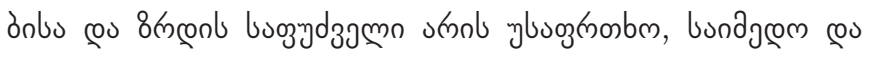

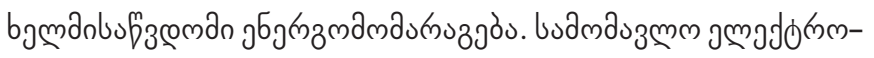

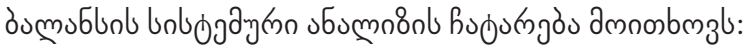

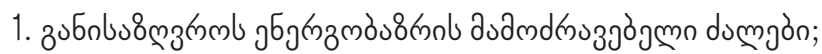

2. zus bumno

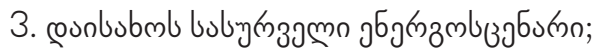

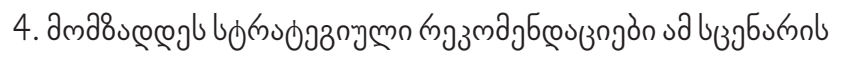
зubbminzogmajobluonzol.

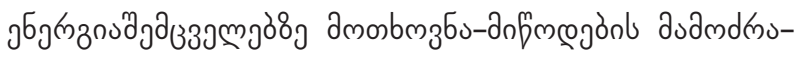

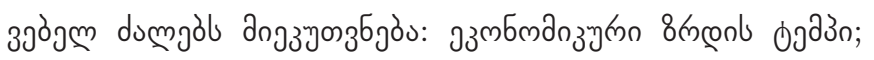

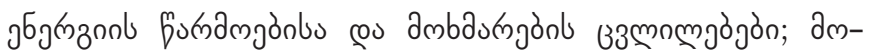
мbmzб

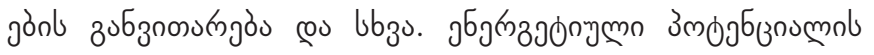
z дамy

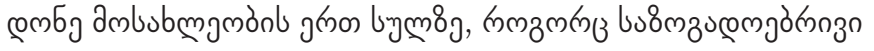
उ ऊrmz

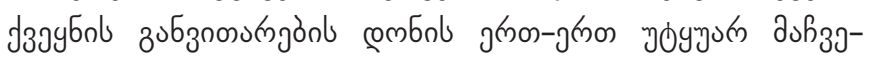

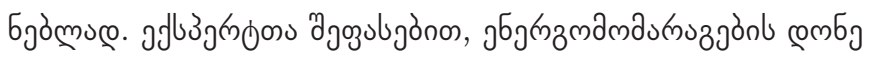

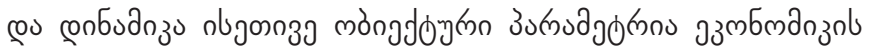

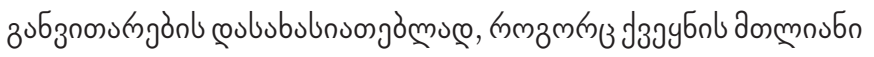

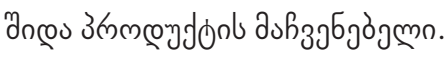

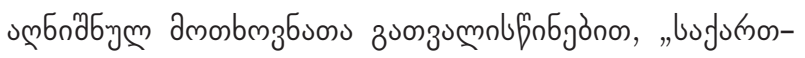

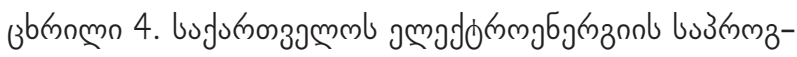

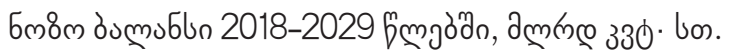

\begin{tabular}{|c|c|c|c|c|c|c|}
\hline \multirow[b]{2}{*}{ Emgòn } & \multirow[b]{2}{*}{$83^{6} g$ mogrou } & \multicolumn{3}{|c|}{ ason anmonls } & \multirow[b]{2}{*}{ ambà mjò } & \multirow[b]{2}{*}{ o adb3mmon } \\
\hline & & 3 & ol & 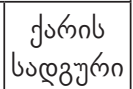 & & \\
\hline 2018 & 13,34 & 11,13 & 2,12 & 0,093 & 13,00 & 0,34 \\
\hline 2019 & 14,47 & 11,85 & 2,53 & 0,093 & 13,65 & 0,82 \\
\hline 2020 & 15,95 & 12,46 & 3,40 & 0,093 & 14,33 & 1,62 \\
\hline 2021 & 18,03 & 15,10 & 2,84 & 0,093 & 15,05 & 2,98 \\
\hline 2022 & 20,35 & 17,91 & 2,34 & 0,093 & 5,80 & 4,55 \\
\hline 2023 & 9 & 36 & 2,43 & 093 & 6,59 & 5,30 \\
\hline 2024 & 23,20 & 20,56 & 2,55 & 0,093 & 17,42 & 5,79 \\
\hline 2025 & 26,34 & 23,63 & 2,61 & 0,093 & 18,29 & 8,05 \\
\hline 2026 & 27,82 & 24,63 & 3,09 & 0,093 & 19,21 & 8,61 \\
\hline 2027 & 29,57 & 26,28 & 3,20 & 0,093 & 20,17 & 9.41 \\
\hline 2028 & 29.92 & 26.62 & 3.20 & 0.093 & 21.17 & 8,74 \\
\hline 2029 & 32.26 & 28.97 & 3.20 & 0.093 & 22.23 & 10.03 \\
\hline
\end{tabular}

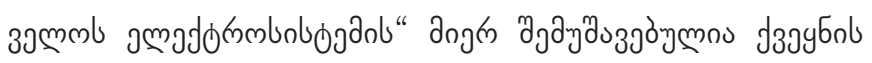

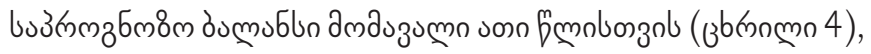

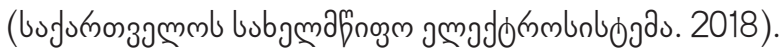

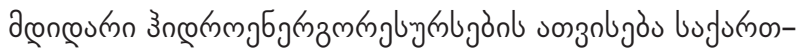

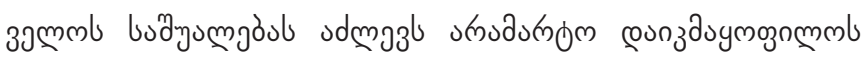

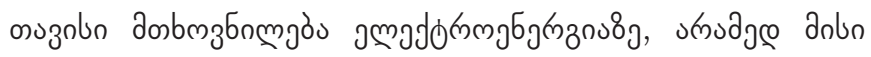
26пдз

\section{esı336s}

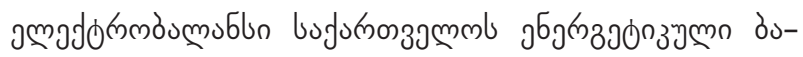
mublin dosszumn zaduœ ornn r.

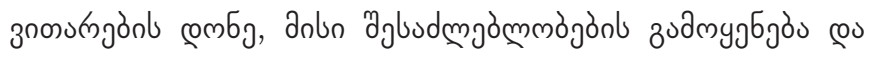

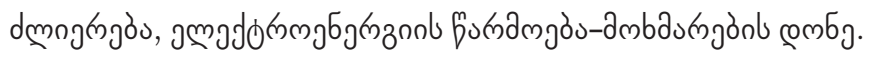

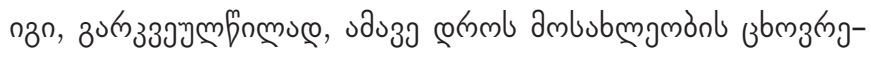

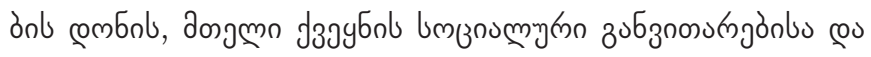

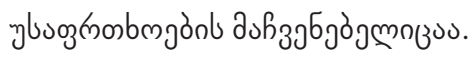

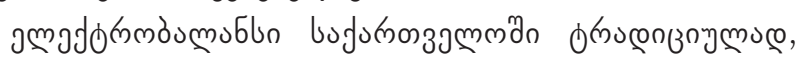

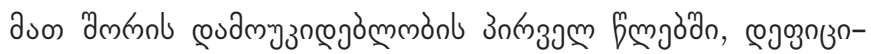

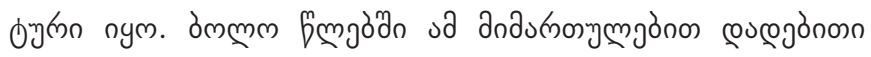

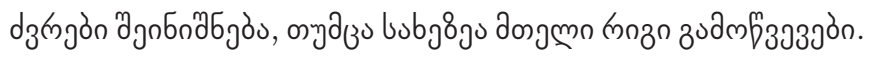

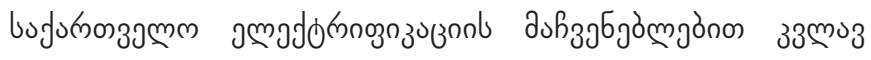

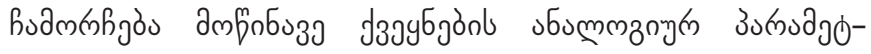

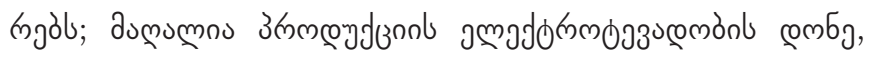

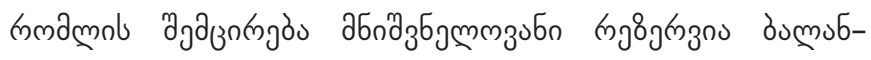

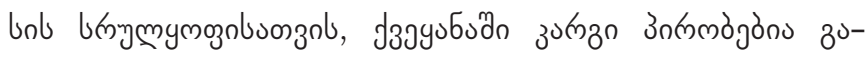




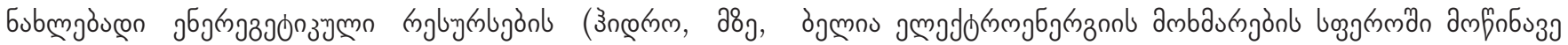

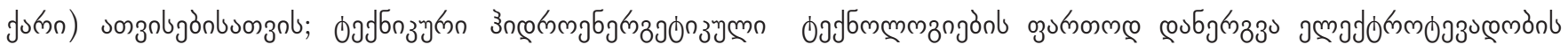

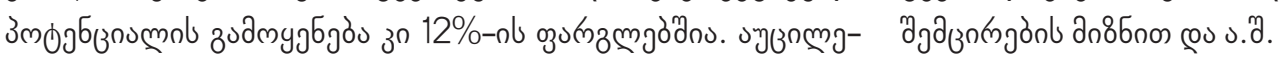

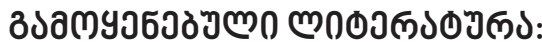

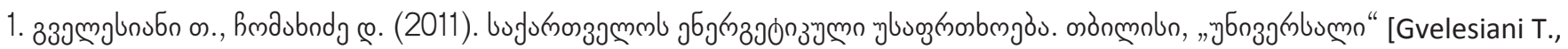
Chomakhidze D. (2011). Georgia ss energy security. Tbilisi, «Universali“. In Georgian].

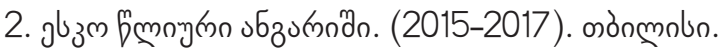

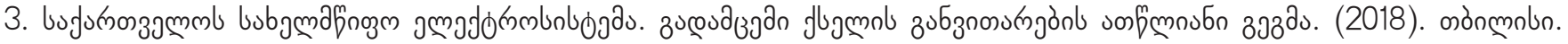
[Georgian State Electro system Transmission network development ten year plan. (2018). Tbilisi. In Georgian].

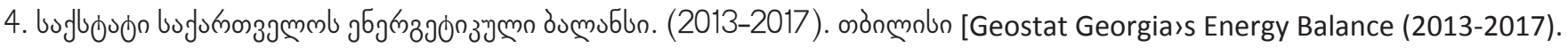
Tbilisi. In Georgia].

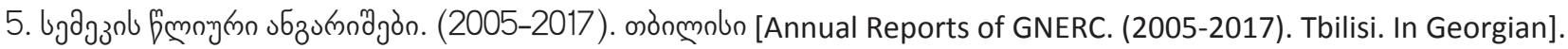

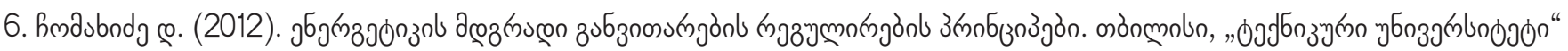
[Chomakhidze D. (2012). Principles of regulating sustainable development of energy. Tbilisi, «Technical University». In Georgian].

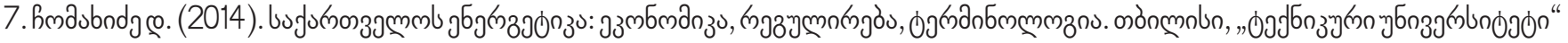
[Chomakhidze D. (2014). Georgia Energy: Economics, Regulation, Terminology. Tbilisi, «Technical University». In Georgian].

8. Chomakhidze D. Tskhakaia K., others. Electricity balance of Georgia: trends and prospects, Energy Procedia (2018) pp. 581587. DOI: 10.1016/j.egypro.2018.07.074.

9. Chomakhidze D., Shengelia G. (2017). Energy Complex of Georgia. Lap, Lambert Academic Publishing.

10. Key World Energy Statistics. (2016). 


\section{THE TRENDS OF DRAWING UP THE ELECTRICITY BALANCE OF GEORGIA}

\section{DEMUR CHOMAKHIDZE}

Doctor of Economics, Professor

Georgian Technical University

Academician of the Academy of Economic Sciences of Georgia, Georgia

demurchomakhidze@yahoo.com

\section{KETEVAN TSKHAKAIA}

Candidat of Technical Science, Associate Professor

Akaki Tsereteli State University, Georgia

ketevan.tskhakaia@gmail.com

\section{KEYWORDS: BALANCE, ELECTRICITY, EXPORT-IMPORT, POWER PLANTS, THERMAL PLANTS.}

For citation: Chomakhidze D., Tskhakaia K. (2019), The Trends Of Drawing Up The Electricity Balance Of Georgia, Globalization And Business, №7, pp. 117-122. (In Georgian). https://doi.org/10.35945/gb.2019.07.014

\section{SUMMARY}

The paper dwells on the analysis of electricity balance of Georgia in the years of 2005-2017 in accordance with the parameters such as electricity generation-consumption and exports-imports. Significant attention was paid to the structural development of electricity generation and consumption. The paper also addresses the issues of exportsimports with neighboring countries. Electricity exports and imports in Georgia is characterized by changing dynamics. Over the past 17 years, imports have grown by $39,3 \%$. It has been highlighted that the electricity balance in the years of
2016-2017 differs substantially from the electricity balance of previous years that is due to the operation of wind power plant, which just in 2017 generated 87,8 million kWh, representing $1 \%$ of generated electricity.

The paper highlights that the level of electric intensity of production in Georgia is still high, the reduction of which is considerable room for improvement in the electricity balance; there is need for development of renewable energy resources (hydro, solar, wind), placing greater reliance on advanced technologies in the field of electricity consumption and so on. 